\title{
Chronic myeloid leukemia patients' adherence to peroral tyrosine kinase inhibitors compared with adherence as estimated by their physicians
}

This article was published in the following Dove Press journal:

Patient Preference and Adherence

24 November 2014

Number of times this article has been viewed

\author{
Meri Kekäle' \\ Kimmo Talvensaari ${ }^{2}$ \\ Perttu Koskenvesa ${ }^{3}$ \\ Kimmo Porkka ${ }^{3}$ \\ Marja Airaksinen' \\ 'Clinical Pharmacy Group, \\ Division of Pharmacology and \\ Pharmacotherapy, Faculty of Pharmacy, \\ University of Helsinki, Helsinki, \\ Finland; ${ }^{2}$ Medical Center Mehiläinen, \\ Nummela, Finland; ${ }^{3} \mathrm{Hematology}$ \\ Research Unit Helsinki, Department \\ of Medicine, Helsinki University \\ Central Hospital and University \\ of Helsinki, Helsinki, Finland
}

Correspondence: Meri Kekäle University of Helsinki, Parantolankatu I0, 05800 Hyvinkää, Finland

Tel +358407 737750

Email meri.kekale@helsinki.fi
Purpose: To evaluate chronic myeloid leukemia (CML) patients' adherence to peroral tyrosine kinase inhibitors in Finland and to compare this with adherence as estimated by their physicians. Other aspects studied included how patients' knowledge of the disease and its treatment influence adherence.

Materials and methods: A total of 120 CML patients were contacted between June 2012 and September 2013 in eight secondary or tertiary care hospitals in Finland. Of these, 86 participated in the study. This covers approximately $20 \%$ of all Finnish CML patients. The mean age was 57.8 years and $52 \%$ were male. Of the patients, $79.1 \%$ were using imatinib, $10.5 \%$ dasatinib, and $10.5 \%$ nilotinib. The patient-reported adherence (experienced adherence) was evaluated using the eight-item Morisky Medication Adherence Scale (MMAS). In addition, the treating physicians were asked to give their subjective opinion on their patients' adherence (observed adherence). The experienced adherence was compared with the observed adherence using a three-level rating system (high, medium, low). All patients were personally interviewed and their demographic data collected. The statistical analysis of the data was based on descriptive statistics presented as frequencies, percentages, means, and medians. The kappa coefficient was calculated between the patient's and the doctor's assessment of adherence.

Results: A total of $23 \%(20 / 86)$ of the patients were fully adherent according to the MMAS, while physicians evaluated $94 \%(80 / 86)$ of the patients as fully adherent. The physicians' estimate was too optimistic in $73 \%$ of cases. The discrepancy was confirmed by a kappa value of -0.004 . The patients' knowledge of the disease and its treatment was poor in all adherence levels.

Conclusion: The patient-reported adherence to tyrosine kinase inhibitor treatments in Finland was found to be the same as that found in the majority of previous studies. However, there seems to be a very weak agreement between the patient's and the physician's assessment of adherence. This study suggests that physicians overestimate the adherence of CML patients and base their assessment primarily on the clinical treatment response.

Keywords: chronic myeloid leukemia, medication adherence, tyrosine kinase inhibitors, Morisky 8-item Medication Adherence Scale, physician's assessment, knowledge of the disease

\section{Introduction}

Chronic myeloid leukemia (CML) is a disease characterized by a consistent chromosomal abnormality (the Philadelphia $[\mathrm{Ph}]$ chromosome), which carries a unique fusion gene called $B C R-A B L 1 .{ }^{1} \mathrm{CML}$ has three stages: chronic; accelerated; and blast crisis. Approximately $85 \%$ of CML cases are diagnosed in the chronic phase. ${ }^{2}$ In the absence of medication, the disease progresses from chronic to accelerated to the blast phase and is fatal. 
After the invention of the first tyrosine kinase inhibitor (TKI), imatinib $\left(\right.$ Glivec $\left.^{\circledR}\right), 10$ years ago, CML has been transformed from a fatal illness to a chronic one. ${ }^{1}$ During the last 5 years, second-generation TKIs (dasatinib, nilotinib) have been developed, giving physicians more options to treat CML patients. Prior to the introduction of TKIs, the median survival for patients with CML was approximately 4 years. ${ }^{3}$ The progression of the disease has slowed: nowadays only $7 \%$ of patients progress to the accelerated or blast phases, ${ }^{4}$ and overall survival rates are almost $90 \%$ (89\% at 5 years and $88 \%$ at 6 years). ${ }^{5,6}$ In practice, this means that the disease is currently managed as long-term oral maintenance therapy by the patient at home. This is a dramatic improvement compared with the time before the launch of modern TKIs.

Regular and adequate dosing is required to achieve therapeutic outcomes by TKI therapy, though adherence is critical. ${ }^{7}$ Adherence has been defined by the World Health Organization as "the extent to which a person's behavior taking medication, following a diet, and executing lifestyle changes - corresponds with agreed recommendations from a healthcare provider". ${ }^{8}$ Many studies have demonstrated that poor medication adherence is related to increased morbidity, mortality and health care costs. ${ }^{7,910}$ The nonadherence rates for oral anticancer therapies vary, and the average rate has been estimated as $21 \% .{ }^{11}$ It is known that adherence to oral CML therapy declines as the duration of the treatment increases. ${ }^{12,13}$

Adherence to imatinib has been reported to range from $60 \%-97 \% .^{14,15}$ A Brazilian study applying the patientadministered 4-item Morisky Medication Adherence Scale (MMAS) showed an even lower adherence rate of 51\%57\%. ${ }^{16}$ Adherence to second-line therapies with dasatinib and nilotinib has varied. ${ }^{12,17}$

Variations in the reported adherence rates to TKIs in CML treatment may be due to variations in the assessment methods. ${ }^{3}$ Most of the studies have applied patient surveys ${ }^{7,9,15-17}$ or register-based adherence estimates. ${ }^{12}$ Little is known about how treating physicians estimate their CML patients' adherence to TKI therapies. No studies have compared patients' and physicians' adherence assessments to show how well physicians are aware of potential adherence problems. This information is essential because patients self-manage their TKI therapies at home. The aim of this study was to evaluate adherence to TKI medication (imatinib, dasatinib, nilotinib) in adult CML patients in Finland who had been on TKI medication for at least the last 6 months before the assessment. Particularly, we were interested in comparing the adherence reported by patients with the adherence assessed by their physicians (experienced versus observed adherence). Other aspects studied included how patients' knowledge of the disease and its treatment influence adherence.

\section{Materials and methods}

The study period was from June 2012-September 2013. All Finnish secondary and tertiary care hospitals (number $[n]=17$ ) treating CML patients were invited to participate in the study. Eight of them agreed to participate. The patients were recruited for this study by the physicians treating CML patients in these hospitals.

The exact number of CML patients in Finland is not known, since there is no register covering all the patients. However, almost all CML patients are on TKI medication, which is fully reimbursed by the Public Social Insurance covering the entire population. ${ }^{18}$ Reimbursements are managed by the Social Insurance Institution, Kela, which is supervised by the government. In 2012, the number of CML patients receiving reimbursement for TKI treatment was 450 (335 received imatinib, 51 dasatinib, and 64 nilotinib). Based on this, we estimate that there are approximately 450 CML patients in Finland. CML medication costs KELA $€ 15$ million a year.

\section{Tools to evaluate adherence}

\section{Experienced adherence}

Patient-reported adherence (experienced adherence) was evaluated by using the eight-item MMAS. ${ }^{19}$ The MMAS is a structured questionnaire validated to estimate adherence to treatment and is widely used in chronic diseases. It was developed from Morisky's previous four-item scale,$^{20}$ which has been used in two published CML adherence studies. ${ }^{16,21}$ The eight-item scale consists of seven questions with "yes" or "no" alternatives, and one item (the last one) features a 5-point Likert scale (Table S1). The MMAS evaluates items addressing the circumstances surrounding adherence behavior. ${ }^{19}$ Each item measures a specific medication-taking behavior and is not a determinant of adherence behavior. The MMAS scores can range from $0-8$ and have been classified into three levels of adherence: high adherence (score 8); medium adherence (score 6-7.75); and low adherence $($ score $<6) .{ }^{19}$

As this is the first time MMAS has been used in a Finnishspeaking patient group, the instrument was translated into Finnish using a method that assures its face validity. ${ }^{22}$ The MMAS was first translated from English into Finnish. The Finnish version was then independently translated by another 
translator back into English. If significant differences were apparent, they were discussed until equivalence between both versions was achieved.

\section{Observed adherence}

In addition to the experienced adherence, we asked the treating physicians to give their subjective opinion on their patients' adherence, which we defined as the observed adherence. If the patient did not have a long-term doctor relationship, the questions were put to the physician who had treated the patient during the patient's last appointment at the hospital. The physician's opinion was rated to represent the following: 1) "high adherence" if the physician thought the patient was taking the medication as prescribed (selection of item 1);2) "medium adherence" if there might be some problems with medication taking (selection of item 2); and 3) "low adherence" if the doctor thought the patient had severe problems and was nonadherent (selection of item 3 ) (Table S2). The experienced adherence was compared with the observed adherence using the three-level rating system (high, medium, low) described above.

\section{Patient interviews}

All patients were interviewed in person and the MMAS questions were asked at the beginning of the interview. Interviews were digitally recorded, transcribed verbatim, and analyzed. Each patient's demographic data were collected during the interview as follows: sex; age; marital status; education; occupation; employment status; time from CML diagnosis; time from diagnosis to the start of TKI treatment; TKI medication; dose; line; duration of usage; comorbidities; other medications; medication review; dosing time; number of medical doctor (MD) visits during the last 12 months; MD visits during the first year after TKI start; nurse contacts in 12 months; nurse contacts during the first year after TKI start; phone contacts in the last 12 months; and days in hospital/hospitalization for any reasons after CML diagnosis. The patient's knowledge of the disease and TKI treatment was evaluated by asking five questions (Table S3). Every correct answer scored 1 point (ie, the total score ranged from $0-5$ points).

At the time of the interview, the therapeutic response to TKI was assessed according to the patient's individual follow-up schedule. All patients recruited gave their written consent. The study protocol was approved by each participating hospital's Ethical Committee.

The statistical analysis of the data was based on descriptive statistics calculated as frequencies, percentages, means, and medians. The kappa coefficient was calculated between the patient's and the doctor's assessment of adherence.

\section{Results}

\section{Patient population}

During the 15-month study period (from June 2012-September 2013), 120 patients were contacted in eight hospitals (four tertiary and four secondary care hospitals). A total of 86 patients participated in the study (approximately 20\% of all Finnish CML patients). Twenty-seven patients declined and seven dropped out after the initial agreement because of a deterioration in their general health, or because they had second thoughts. In this patient population, the mean time from CML diagnosis was 5 years (median: 4 years; range: $1-17$ years) (Table 1). The mean age at diagnosis was 53 years (median: 52 years; range: 19-79 years). Patient characteristics are shown in Table 1.

\section{Medication/treatment}

CML medication-related factors are shown in Table 1. Most of the patients (79\%) were receiving imatinib, $11 \%$ dasatinib, and $11 \%$ nilotinib. Most of the patients were on first- $(55 \%)$ or second- $(29 \%)$ line treatment. First-line treatment means that the patients were on the same active substance and dose as when they had started the treatment. Most (84\%) of the patients were taking a single dose, usually in the morning (31\%) or during lunchtime (27\%). None of the patients had a written treatment plan, and only $5 \%(n=4)$ had a list of their medications.

Seventy-six percent of the patients had other diseases (mean: 2; median: 1; range: 0-8) (Table 1). Eight percent of the patients had previously had a diagnosis of at least one other cancer (range: 0-3). Due to these comorbidities, the patients were also using other medications, the mean number of which was 2 (median: 1; range: $0-10$ ).

\section{Response to TKI treatment}

The response to TKI treatment was high in this study, with $81 \%$ of the patients showing an optimal response to their treatment according to European LeukemiaNet 2013 recommendations. ${ }^{23}$ One-third of the patients had shown a major molecular response $\left(\mathrm{MR}^{3.0}\right)$. The proportion of patients with $\mathrm{MR}^{5.0}$ was very high $(23 \%)$. The correlation between the adherence rate and treatment response is shown in Table 2.

\section{Knowledge of the disease}

The CML patients' knowledge of the disease and its treatment was poor, as they scored on average 1 point out of 
Table I Characteristics of the CML patients on TKI medication involved in the study $(n=86)$

\begin{tabular}{|c|c|c|}
\hline \multicolumn{3}{|l|}{ Variables } \\
\hline \multirow[t]{2}{*}{ Sex, n (\%) } & Male & $45(52.3)$ \\
\hline & Female & $4 \mathrm{I}(47.7)$ \\
\hline \multirow[t]{3}{*}{ Age $^{a}$ (years) } & Mean (SD) & $57.8(12.1)$ \\
\hline & Median & 59.0 \\
\hline & Range & $25.0-83.0$ \\
\hline \multirow[t]{3}{*}{ Age at diagnosis (years) } & Mean (SD) & $52.7(12.3)$ \\
\hline & Median & 52.0 \\
\hline & Range & 19.0-79.0 \\
\hline \multirow[t]{3}{*}{ Time from diagnosis (years) } & Mean (SD) & $5.1(3.7)$ \\
\hline & Median & 4.0 \\
\hline & Range & $0.5-17.0$ \\
\hline \multicolumn{3}{|l|}{ TKI medication-related factors } \\
\hline \multirow[t]{3}{*}{ TKI medication, $\mathrm{n}(\%)$} & Imatinib & $68(79.1)$ \\
\hline & Dasatinib & $9(10.5)$ \\
\hline & Nilotinib & $9(10.5)$ \\
\hline \multirow[t]{4}{*}{ Line, $n(\%)$} & First & $47(54.7)$ \\
\hline & Second & $25(29.1)$ \\
\hline & Third & $13(15.1)$ \\
\hline & Fourth & $\mathrm{I}(\mathrm{I} .2)$ \\
\hline \multirow[t]{2}{*}{ Number of TKI doses per day, $\mathrm{n}(\%)$} & One & $72(83.7)$ \\
\hline & Two & $14(16.3)$ \\
\hline \multicolumn{3}{|l|}{ Visits and contacts with health personnel } \\
\hline \multirow[t]{3}{*}{ MD visits in the last 12 months } & Mean (SD) & $2.2(1.8)$ \\
\hline & Median & 2.0 \\
\hline & Range & $0-12$ \\
\hline \multirow{3}{*}{$\begin{array}{l}\text { MD visits during the first year after } \\
\text { diagnosis }\end{array}$} & Mean (SD) & $4.1(1.5)$ \\
\hline & Median & 4 \\
\hline & Range & $1-12$ \\
\hline \multirow{3}{*}{ Phone contacts in the last 12 months } & Mean (SD) & $0.7(1.7)$ \\
\hline & Median & 0 \\
\hline & Range & $0-12$ \\
\hline \multicolumn{3}{|l|}{ Adherence aids } \\
\hline \multirow[t]{2}{*}{ Written treatment plan, $\mathrm{n}(\%)$} & No & $86(100)$ \\
\hline & Yes & 0 \\
\hline \multirow[t]{2}{*}{ List of medications, $n$ (\%) } & No & $82(95.3)$ \\
\hline & Yes & $4(4.7)$ \\
\hline \multirow[t]{2}{*}{ Pill dispenser, n (\%) } & No & $69(80.2)$ \\
\hline & Yes & $17(19.8)$ \\
\hline \multirow[t]{2}{*}{ Mobile phone reminder, $\mathrm{n}(\%)$} & No & $76(88.4)$ \\
\hline & Yes & $10(11.6)$ \\
\hline \multirow[t]{6}{*}{ Knowledge total score, $\mathrm{n}(\%)$} & 0 & $28(32.6)$ \\
\hline & 1 & $30(34.9)$ \\
\hline & 2 & $15(17.4)$ \\
\hline & 3 & $7(8.1)$ \\
\hline & 4 & $4(4.7)$ \\
\hline & 5 & $2(2.3)$ \\
\hline
\end{tabular}

Note: at the time of adherence evaluation.

Abbreviations: CML, chronic myeloid leukemia; TKI, tyrosine kinase inhibitor; $\mathrm{n}$, number; SD, standard deviation; MD, medical doctor.

the maximum 5 points (Table 1). One-third of the patients scored nothing from the questions, which means they did not understand their disease, how the medication works, or the consequences of not taking the medicine as prescribed.
Only $2 \%(\mathrm{n}=2)$ of the patients scored the maximum 5 points. There was no difference in patients' knowledge in different adherence levels.

\section{Physician-related characteristics}

Thirteen physicians were involved in the study. Twelve of them were specialists in hematology and one in internal medicine. The patients had had, on average, two MD visits during the last 12 months (median: 2; range: 0-12). There was no difference in the number of MD visits between different adherence levels. Two-thirds of the patients had a long-term relationship with their physician (67\%), while the other $33 \%$ reported that their physician changed every second visit (Table 2).

\section{Experienced and observed adherence}

Despite the high molecular response rates to TKI treatment, adherence according to MMAS was not good in most of the patients: less than a quarter $(23 \%)$ of the patients showed high adherence; 56\% exhibited medium adherence; and $21 \%$ showed low adherence (Table 2). The distribution of the eight items indicating nonadherent behaviors according to the MMAS is shown in Figure 1. Unintentional nonadherence was common, particularly forgetting to take the medication ( $48 \%$ of patients). One-fifth of the patients had sometimes forgotten to bring along their medication when leaving home or travelling. Patients in the present study had a low level of intentional nonadherence behavior. Stopping taking the medication when feeling worse after drug administration without telling the doctor was rare: only $11 \% \mathrm{had}$ done so. Twenty-two percent of the patients reported taking their medication every day to be a real inconvenience.

There was a considerable difference between observed and experienced adherence: $94 \%$ of the patients were highly adherent according to the physicians' assessment. The kappa coefficient between the patients' and physicians' assessment of adherence was extremely low $(-0.004)$, indicating that there was no agreement between the two assessments. Five percent were assessed as medium adherent by physicians and only $1 \%$ as low adherent. Compared to the patientreported adherence, the physicians' assessments were too optimistic in $73 \%$ of cases, realistic in $25 \%$ of cases, and pessimistic in $2 \%$ of cases. In 17 cases $(20 \%$ of the total patient population), the physician had assessed the patient as highly adherent, whereas the MMAS scored the patient as low adherent. In 45 cases ( $52 \%$ of the patients), patients who were medium adherent according to the MMAS were assessed by the physician as highly adherent. 
Table 2 Adherence of CML patients to TKI treatment as measured by the MMAS scale $(n=86)$

\begin{tabular}{|c|c|c|c|c|}
\hline & MMAS 8 (high) & MMAS 6-7.75 (medium) & MMAS < 6 (low) & Total, n (\%) \\
\hline Total, n (\%) & $20(23)$ & $48(56)$ & $18(2 \mid)$ & $86(100)$ \\
\hline Age (years), median (range) & $62(46-82)$ & $58(3 \mid-83)$ & $49(25-82)$ & \\
\hline \multicolumn{5}{|l|}{ Sex, n (\%) } \\
\hline Male & II (25) & $23(52)$ & $10(23)$ & $44(100)$ \\
\hline Female & $9(22)$ & $25(61)$ & $8(20)$ & $42(100)$ \\
\hline \multicolumn{5}{|l|}{ Time from diagnosis (years) } \\
\hline Mean (range) & $4(I-I I)$ & $6(1-17)$ & $5(1-15)$ & \\
\hline Median & 3 & 6 & 3 & \\
\hline \multicolumn{5}{|l|}{ Medication, n (\%) } \\
\hline Imatinib & $16(24)$ & $40(59)$ & $12(18)$ & $68(100)$ \\
\hline Dasatinib & $I(10)$ & $6(56)$ & $3(33)$ & $9(100)$ \\
\hline Nilotinib & $3(33)$ & $3(33)$ & $3(33)$ & $9(100)$ \\
\hline \multicolumn{5}{|l|}{ Line, n (\%) } \\
\hline First & $12(26)$ & $26(55)$ & $9(19)$ & $47(100)$ \\
\hline Second & $5(20)$ & $12(48)$ & $8(32)$ & $25(100)$ \\
\hline Third & $3(23)$ & $10(77)$ & 0 & $13(100)$ \\
\hline Fourth & 0 & 0 & $\mathrm{I}(100)$ & $I(100)$ \\
\hline \multicolumn{5}{|c|}{ Long-term doctor relationship, n (\%) } \\
\hline Yes & $15(26)$ & $31(53)$ & $12(2 \mid)$ & $58(100)$ \\
\hline No & $5(18)$ & $17(61)$ & $6(21)$ & $28(100)$ \\
\hline \multicolumn{5}{|l|}{ Treatment response, $\mathrm{n}(\%)$} \\
\hline $\mathrm{PCyR}^{\mathrm{a}}$ & 0 & $3(100)$ & 0 & $3(100)$ \\
\hline $\mathrm{CCyR}$ & $3(23)$ & $10(77)$ & 0 & $13(100)$ \\
\hline$M R^{3.0}$ & $9(27)$ & $14(4 \mid)$ & II (32) & $34(100)$ \\
\hline $\mathrm{MR}^{4.0}$ & $4(44)$ & $4(44)$ & $\mathrm{I}(\mathrm{I} 2)$ & $9(100)$ \\
\hline$M R^{4.5}$ & 0 & $7(100)$ & 0 & $7(100)$ \\
\hline $\mathrm{MR}^{5.0}$ & $4(20)$ & $10(50)$ & $6(30)$ & $20(100)$ \\
\hline
\end{tabular}

Notes: Cytogenetic responses: Partial cytogenetic response (PCyR), Ph+ metaphases I-35\%. Complete cytogenetic response (CCyR), no Ph+ metaphases or less than I\% BCR-ABLI positive nuclei by I-FISH. Molecular responses: $M^{3.0}=$ major molecular response $=B C R-A B L I<0.0 I \%(I S) ; M R^{4.0}=B C R-A B L I \leq 0.0 I \%(I S) ; M R^{4.5}=B C R-$ $A B L I \leq 0.0032 \%$ (IS); MRR ${ }^{5.0}=B C R-A B L I \leq 0.001 \%$ (IS). ${ }^{23}$ Response is assessed with a standardized real quantitative polymerase chain reaction and/or cytogenetics at 3 months, 6 months, and 12 months. BCR-ABLI transcript levels $\leq 10 \%$ at 3 months, $<1 \%$ at 6 months, and $\leq 0.1 \%$ from 12 months onward define optimal response, whereas $>10 \%$ at 6 months and $>1 \%$ from 12 months onward define failure. PCyR at 3 months and CCyR from 6 months onward define optimal response, whereas no CyR (PhI+ $>95 \%$ ) at 3 months, less than PCyR at 6 months, and less than CCyR from 12 months onward define failure. ${ }^{23}$ a Ph+ metaphases I- $35 \%$. ${ }^{b} \mathrm{No} P h+$ metaphases or less than I\% BCR-ABLI positive nuclei by I-FISH.

Abbreviations: CML, chronic myeloid leukemia; TKI, tyrosine kinase inhibitor; MMAS, 8-item Morisky Medication Adherence Scale; n, number; CyR, cytogenetic response; $\mathrm{MR}$, molecular response; $\mathrm{Ph}+$, Philadelphia chromosome-positive; IS, international scale; I-FISH, interphase fluorescence in situ hybridization.

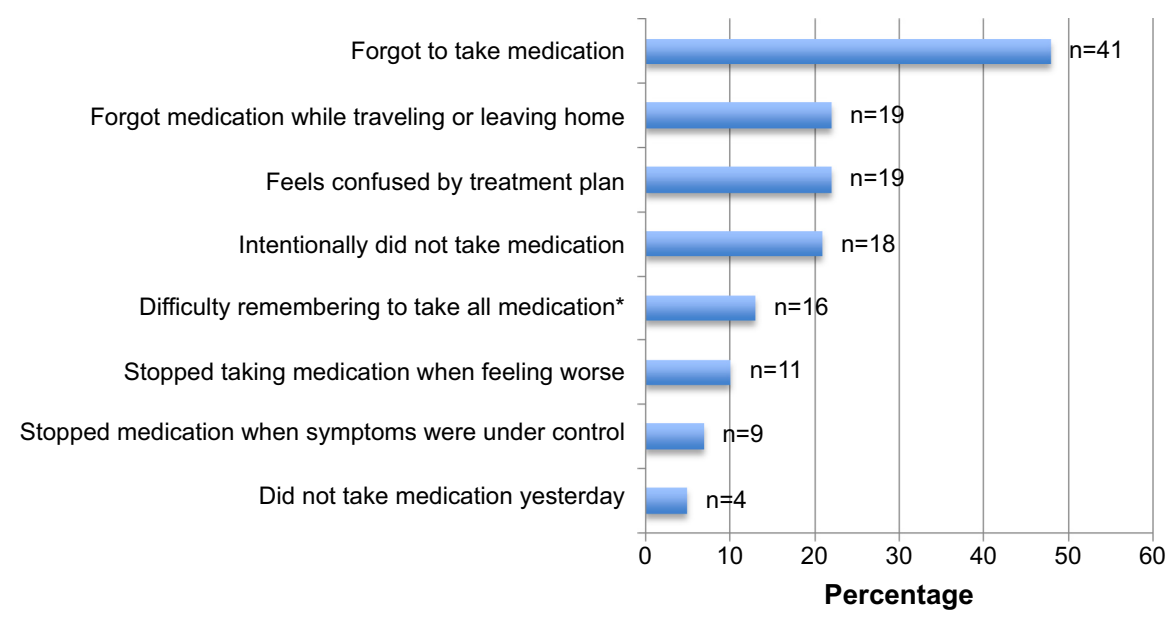

Figure I Nonadherent behaviors of CML patients on TKI medication as measured by items included in the MMAS ( $n=86$ ).

Note: *Item contains a 5-point Likert scale (once in a while, sometimes, usually, all the time $=<$ I point; included in the figure).

Abbreviations: n, number; CML, chronic myeloid leukemia; TKI, tyrosine kinase inhibitor; MMAS, 8-item Morisky Medication Adherence Scale. 


\section{Discussion}

The present study employs a novel approach to adherence assessment by CML patients and their physicians. We found the same, quite low patient-reported adherence to TKI treatments that has been found in most previous studies. ${ }^{71,14-16}$ However, in most of these cases with low patient-experienced adherence, physicians estimated adherence to be high. Consequently, our findings suggest that there is a discrepancy between patient-experienced and physicianobserved adherence. This finding was confirmed by the low kappa value for inter-rater reliability.

One reason for this discrepancy in adherence estimates by patients and their physicians may be clinical treatment outcomes. Despite the low patient-reported adherence rate assessed by MMAS, most of the patients ( $81 \%$ ) had an optimal molecular response to their treatment according to European LeukemiaNet (ELN) 2013 Guidelines. ${ }^{23}$ Consequently, we assume that good clinical treatment outcomes were the reason why the physicians gave a higher estimate of adherence than the patients. On this basis, we also assume that in many cases, physicians' estimates of adherence are based primarily on the evaluation of treatment outcomes, as indicated by clinical laboratory tests. Our findings also suggest that patient-experienced adherence can be low, even though the clinical treatment response is optimal.

According to our findings, physicians seem to be too optimistic in assessing their patients' adherence. Previous studies with other patient groups and medications show that physicians are inaccurate in assessing adherence and judging which patients are adherent and which are not. ${ }^{15,24-26}$ When estimating the degree of adherence for any particular patient group, physicians have been shown to be accurate only $10 \%-40 \%$ of the time, for both medication and other treatments. ${ }^{27-29}$ In the Belgian ADAGIO study ${ }^{15}$ physicians believed that, on average, $93 \%$ of CML patients on imatinib were adherent during the first month after diagnosis and that $87 \%$ were adherent after 1 year of treatment.

Our findings indicate that most of the CML patients involved in the study were willing to take their TKI medicines as prescribed. Thus, intentional nonadherence was rare according to the MMAS measures used. The most common reason was forgetting to take the TKI medicine. This indicates that CML patients may need support and practical aids to help them manage their medications in everyday life. As part of this, they should be better involved in their care by providing them with a medication list and therapeutic plan. According to our study findings, a few of the patients had a medication list and none knew about their therapeutic plan. These simple tools could also increase patients' knowledge of CML as a disease and its treatment, which was found to be poor in our study. Solving these medication managementrelated issues may require physicians to be more aware of a range of factors related to adherence and self-management of long-term diseases, such as CML, requiring patients' active involvement.

Simple measures, such as the MMAS, might be helpful in identifying some of the adherence problems in clinical practice. MMAS is a short and easily used tool applicable to routine practice. More important than the numeric value of adherence is the information that the eight items provide about medicine use. The MMAS's eight items indicate the intentional and unintentional reasons for nonadherence and could help physicians to evaluate adherence, identify adherence problems, and discuss them with the patient. It is important to train physicians and other health care providers about adherence and its role in managing medication use in clinical practice.

CML has changed from a fatal to a chronic disease during the last decade. Nowadays, the patient is fully responsible for managing the treatment at home. This change presumes a new type of cooperation partnership between the physician and the patient. The physician is the medical expert when it comes to the illness and its medication, while the patient best understands his/her everyday life with the disease and its treatment. ${ }^{30}$ It is important to understand the root causes of patients' nonadherence in order to encourage them to take responsibility for their own treatment. Further research is needed to understand these changes in the medication management processes of CML patients arising from current advances in pharmacotherapies.

The present study has some limitations. The study population covered $20 \%$ of the Finnish CML patient population and involved 13 physicians specialized in CML care. Due to the possibility of biasing factors, our results do not necessarily reflect the complete picture of TKI adherence in Finland. There may possibly be more nonadherent patients declining during the study enrolment period, thus resulting in increased selection bias. However, the highly standardized data gathering procedure and personal interviews conducted by one person meant that there was no interobserver bias. On the other hand, the method used to obtain physicians' subjective assessments of their patients' adherence is not well standardized and includes the risk of interobservation bias. In clinical practice, however, the high risk of a subjective overestimation of TKI adherence in individual patients is more relevant. 
The eight-item MMAS shows three different adherence rates. The patients who have the rating 2 or 3 (medium or low) are not "fully adherent". In our study, this portion was $77 \%$ of the patients. The patient needs to score all 8 points to be highly adherent by the MMAS. This might influence the results. The MMAS has not been specially developed for the evaluation of medication adherence in CML patients, although it is widely used in other chronic illnesses. ${ }^{19,20}$ It would be interesting to validate a CML-specific adherence scale in the future. Almost all of the physicians' assessments were "high adherence", which caused bias in the comparison between experienced and observed adherence. We were, therefore, unable to perform any statistical analysis between these two assessments. This should be addressed in future studies.

\section{Conclusion}

The patient-reported adherence to TKI treatments in Finland was found to be the same as that found in the majority of previous studies, with $21 \%$ having poor adherence as measured by the MMAS. However, there seems to be a very weak agreement between the patients' and the physicians' assessment of adherence, physicians having a tendency to overestimate adherence in CML patients. This tendency may be related to the finding that physicians based their adherence estimation primarily on the patients' clinical treatment response. Despite a good clinical response, patients may lack knowledge of CML as a disease and its treatment with TKIs, as well as access to a treatment plan and medication list.

\section{Acknowledgments}

We are grateful to all patients who participated in this study. We would like to thank Professor Kari Remes; Dr Marjut Kauppila, MD, PhD; Dr Venla Terävä, MD, PhD; Dr Hanna Ollikainen, MD, PhD; Dr Sakari Kakko, MD, PhD; Antti Koponen, MD; Dr Maija Mikkola, MD, PhD; Anu Kutila, MD; and Kirsi Launonen, MD, for their help in recruiting the patients for the study. We also thank Dr Lauri Handolin MD, $\mathrm{PhD}$, for his fruitful ideas and for revising the manuscript; and $\mathrm{Dr}$ Tim Söderlund, $\mathrm{MD}, \mathrm{PhD}$, for his valuable ideas with the data analysis. Our thanks go to study nurses, Minna Pajuportti, Raija Aaltonen, Marjut Yrjönen, Ulla Pietilä, Erja Silventoinen, Leena Lahdenmaa, and Riitta Saavalainen, for their help with patient contacts.

\section{Disclosure}

The authors report no conflicts of interest in this work.

\section{References}

1. Goldman JM, Melo JV. Chronic myeloid leukemia - advances in biology and new approaches to treatment. $N$ Engl J Med. 2003;349(15): $1451-1464$.

2. White P, Walker P. Chronic leukemias. In: Skeel R, editor. Handbook of Cancer Chemotherapy. 7th ed. Philadelphia, PA: Lippincott Williams and Wilkins; 2007:497-516.

3. Gater A, Heron L, Abetz-Webb L, et al. Adherence to oral tyrosine kinase inhibitor therapies in chronic myeloid leukemia. Leuk Res. 2012; 36(7):817-825.

4. Wetzler M, Byrd JC, Bloomfield CD. Acute and chronic myeloid leukemia. In: Kasper DL, Braunwald E, Hauser S, Longo D, Jameson JL, Fauci AS. Harrison's Principles of Internal Medicine. 16th ed. New York, NY: McGraw-Hill Companies, Inc.; 2005:631-641.

5. Druker BJ, Guilhot F, O'Brien SG, et al; IRIS Investigators. Five-year follow-up of patients receiving imatinib for chronic myeloid leukemia. N Engl J Med. 2006;355(23):2408-2417.

6. Hochhaus A, O'Brien SG, Guilhot F, et al; IRIS Investigators. Six-year follow-up of patients receiving imatinib for the first-line treatment of chronic myeloid leukemia. Leukemia. 2009;23(6):1054-1061.

7. Marin D, Bazeos A, Mahon FX, et al. Adherence is the critical factor for achieving molecular responses in patients with chronic myeloid leukemia who achieve complete cytogenetic responses on imatinib. J Clin Oncol. 2010;28(14):2381-2388.

8. World Health Organization. Adherence to Long-Term Therapies. Evidence for Action. Geneva, Switzerland: 2003.

9. Ibrahim AR, Eliasson L, Apperley JF, et al. Poor adherence is the main reason for loss of CCyR and imatinib failure for chronic myeloid leukemia patients on long-term therapy. Blood. 2011;117(14): 3733-3736.

10. Darkow T, Henk HJ, Thomas SK, et al. Treatment interruptions and nonadherence with imatinib and associated healthcare costs: a retrospective analysis among managed care patients with chronic myelogenous leukaemia. Pharmacoeconomics. 2007;25(6):481-496.

11. DiMatteo MR. Variations in patients' adherence to medical recommendations: a quantitative review of 50 years of research. Med Care. 2004;42(3):200-209.

12. Ulcickas Yood M, Oliveria SA, Hirji I, Cziraky M, Davis C. Adherence to treatment in patients with chronic myeloid leukemia during a 10-year time period: a medical record review. In: Proceedings of the 53rd ASH Annual Meeting and Exposition; December 10-13, 2011; San Diego, CA; Abstract 1235.

13. Breccia M, Efficace F, Alimena G. Imatinib treatment in chronic myelogenous leukemia: What have we learned so far? Cancer Lett. 2011;300(2):115-121.

14. StCharles M, Bollu VK, Hornyak E, Coombs J, Blanchette CM, DeAngelo DJ. Predictors of treatment non-adherence in patients treated with imatinib mesylate for chronic myeloid leukemia. In: Online Programs and Abstracts for the 51st ASH Annual Meeting and Exposition; December 5-8, 2009; New Orleans, LA. Abstract 2209.

15. Noens L, van Lierde MA, De Bock R, et al. Prevalence, determinants, and outcomes of nonadherence to imatinib therapy in patients with chronic myeloid leukemia: the ADAGIO study. Blood. 2009; 113(22):5401-5411.

16. de Almeida MH, Pagnano KB, Vigorito AC, Lorand-Metze I, de Souza CA. Adherence to tyrosine kinase inhibitor therapy for chronic myeloid leukemia: a Brazilian single-center cohort. Acta Haematol. 2013; 130(1):16-22.

17. Wu EQ, Guerin A, Yu AP, Bollu VK, Guo A, Griffin JD. Retrospective real-world comparison of medical visits, costs, and adherence between nilotinib and dasatinib in chronic myeloid leukemia. Curr Med Res Opin. 2010;26(12):2861-2869.

18. Kaikkonen P, Harsia-Alatalo J. Medicine reimbursement system and approval of medicine prices. In: Finnish Statistics on Medicines. Finnish Medicines Agency Fimea and Social Insurance Institution. Helsinki, Finland: Edita Prima Oy; 2012:71-80. 
19. Morisky DE, Ang A, Krousel-Wood M, Ward HJ. Predictive validity of a medication adherence measure in an outpatient setting. $J$ Clin Hypertens (Greenwich). 2008;10(5):348-354.

20. Morisky DE, Green LW, Levine DM. Concurrent and predictive validity of a self-reported measure of medication adherence. Med Care. 1986;24(1):67-74.

21. Efficace F, Baccarani M, Rosti G, et al. Investigating factors associated with adherence behaviour in patients with chronic myeloid leukemia: an observational patient-centered outcome study. Br J Cancer. 2012;107(6):904-909.

22. Sartorius N, Kuyken W. Translation of health status instruments. In: Orley J, Kuyken W, editors. Quality of Life Assessment: International Perspectives. New York, NY: Springer Berlin Heidelberg; 1994:3-19.

23. Baccarani M, Deininger MW, Rosti G, et al. European LeukemiaNet recommendations for the management of chronic myeloid leukemia: 2013. Blood. 2013;122(6):872-884.

24. Osterberg L, Blaschke T. Adherence to medication. $N$ Engl J Med. 2005;353(5):487-497.

25. Wagner JH, Justice AC, Chesney M, Sinclair G, Weissman S, Rodriguez-Barradas M; VACS 3 Project Team. Patient- and providerreported adherence: toward a clinically useful approach to measuring antiretroviral adherence. J Clin Epidemiol. 2001;54 (Suppl 1): S91-S98.
26. Zeller A, Taegtmeyer A, Martina B, Battegay E, Tschudi P. Physicians' ability to predict patients' adherence to antihypertensive medication in primary care. Hypertens Res. 2008;31(9):1765-1771.

27. Morton A, Riddle R, Buchanan R, Katz D, Birch J. Accuracy in the prediction and estimation of adherence to bracewear before and during treatment of adolescent idiopathic scoliosis. J Pediatr Orthop. 2008; 28(3):336-341.

28. Parker CS, Chen Z, Price M, et al. Adherence to warfarin assessed by electronic pill caps, clinician assessment, and patient reports: results from the IN-RANGE study. J Gen Intern Med. 2007;22(9):1254-1259.

29. Turner BJ, Hecht FM. Improving on a coin toss to predict patient adherence to medications. Ann Intern Med. 2001;134(10):1004-1006.

30. Horne R, Weinman J, Barber N, et al. Concordance, Adherence and Compliance in Medicine Taking: Report for the National Co-ordinating Centre for NHS Service Delivery and Organisation $R \& D$ (NCCSDO). London, UK: National Co-ordinating Centre for NHS Service Delivery and Organisation; 2005. 


\section{Supplementary materials}

Table SI Morisky Medication Adherence Scale (8-item)

You indicated that you are taking medication(s) for your chronic myelogenous leukemia. Individuals have identified several issues regarding their medication-taking behavior and we are interested in your experiences. There is no right or wrong answer. Please answer each question based on your personal experience with your chronic myelogenous leukemia medication. (Please check your response below)

No $=$ I Yes $=0$

I. Do you sometimes forget to take your chronic myelogenous leukemia medication(s)?

2. People sometimes miss taking their medications for reasons other than forgetting. Thinking over the past 2 weeks, were there any days when you did not take your chronic myelogenous leukemia medication(s)?

3. Have you ever cut back or stopped taking your medication(s) without telling your doctor, because you felt worse when you took it?

4. When you travel or leave home, do you sometimes forget to bring along your chronic myelogenous leukemia medication(s)?

5. Did you take your chronic myelogenous leukemia medication(s) yesterday?

6. When you feel like your chronic myelogenous leukemia is under control, do you sometimes stop taking your medication(s)?

7. Taking medication(s) every day is a real inconvenience for some people. Do you ever feel hassled about sticking to your chronic myelogenous leukemia treatment plan?

8. How often do you have difficulty remembering to take all your medication(s)?

(Please circle the correct number)

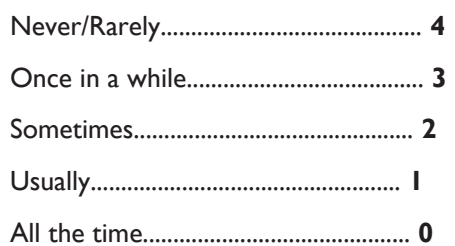

(c) Morisky Medication Adherence Scale (MMAS-8-Item). Use of the CMMAS is protected by US copyright laws. Permission for use is required. A license agreement is available from Donald E Morisky, ScD, ScM, MSPH, Professor, Department of Community Health Sciences, UCLA School of Public Health, 650 Charles E Young Drive South, Los Angeles, CA 90095-1772, USA.

Table S2 Physicians' assessment of chronic myeloid leukemia patients' adherence on the basis of the doctor-patient relationship

I. The patient uses the medication as prescribed and has no problems.

2. Problems in adherence to medication are possible.

3. The patient does not follow the treatment as prescribed. There are problems in medication use. Adherence to the medication is not optimal.

Table S3 Questions to evaluate CML patient's knowledge of the disease and TKI treatment

\section{Question I. What is CML?}

Question 2. What has happened in your body (the cause of CML)?

Question 3. What does the TKI medication do in your body (how does the medication work)?

Question 4. What kind of side effects might the medication cause?

Question 5. (Have you talked with your doctor?) What happens if you skip a dose/stop taking your TKI medication?

Notes: Each question has a value of I point. The total score will range from 0-5.

Abbreviations: CML, chronic myeloid leukemia; TKI, tyrosine kinase inhibitor.

Patient Preference and Adherence

\section{Publish your work in this journal}

Patient Preference and Adherence is an international, peer-reviewed, open access journal that focuses on the growing importance of patient preference and adherence throughout the therapeutic continuum. Patient satisfaction, acceptability, quality of life, compliance, persistence and their role in developing new therapeutic modalities and compounds to optimize

\section{Dovepress}

clinical outcomes for existing disease states are major areas of interest for the journal. This journal has been accepted for indexing on PubMed Central. The manuscript management system is completely online and includes a very quick and fair peer-review system, which is all easy to use. Visit http://www. dovepress.com/testimonials.php to read real quotes from published authors. 